\title{
Detection of Matrix Crack Density of CFRP using an Electrical Potential Change Method with Multiple Probes"
}

\author{
Akira TODOROKI $^{* *}$ and Kazuomi OMAGARI ${ }^{* * *}$ \\ **Department of Mechanical Sciences and Engineering, Tokyo Institute of Technology, \\ 2-12-1, Ookayama, Meguro, Tokyo, Japan \\ E-mail: atodorok@ginza.mes.titech.ac.jp \\ *** Graduate student of Tokyo Institute of Technology, \\ 2-12-1, Ookayama, Meguro, Tokyo, Japan \\ E-mail: komagari@ginza.mes.titech.ac.jp
}

\begin{abstract}
Carbon Fiber Reinforced Plastic (CFRP) laminates are adopted for fuel tank structures of next generation space rockets or automobiles. Matrix cracks may cause fuel leak or trigger fatigue damage. A monitoring system of the matrix crack density is required. The authors have developed an electrical resistance change method for the monitoring of delamination cracks in CFRP laminates. Reinforcement fibers are used as a self-sensing system. In the present study, the electric potential method is adopted for matrix crack density monitoring. Finite element analysis (FEA) was performed to investigate the possibility of monitoring matrix crack density using multiple electrodes mounted on a single surface of a specimen. The FEA reveals the matrix crack density increases electrical resistance for a target segment between electrodes. Experimental confirmation was also performed using cross-ply laminates. Eight electrodes were mounted on a single surface of a specimen using silver paste after polishing of the specimen surface with sandpaper. The two outermost electrodes applied electrical current, and the inner electrodes measured electric voltage changes. The slope of electrical resistance during reloading is revealed to be an appropriate index for the detection of matrix crack density.
\end{abstract}

Key words: CFRP, Composites, Matrix Crack, Electrical Resistance, FEM

\section{Introduction}

A cryogenic fuel tank of a next generation reusable launching vehicle will adopt laminated composite tanks ${ }^{(1)}$. For a fuel tank comprising a laminated Carbon Fiber Reinforced Polymer (CFRP), matrix cracking of the CFRP laminates may cause fuel leakage, and therefore, detection of matrix cracking is required.

A change in electrical resistance of the CFRP structure has been applied to detect damage of a CFRP structure like fiber breakage, fiber-matrix debonding, matrix cracks and delamination ${ }^{(2)-(11)}$. Applied strain and fiber breakages of the CFRP structures are monitored using the electrical resistance changes; electric current is applied to the end of the rectangular specimens ${ }^{(2),(4),(6)}$.

The authors' research group has proposed a delamination monitoring method ${ }^{(12)-}$ 
this research, multiple probes for applying electric current and measuring electric voltage changes are integrated on the single specimen surface using co-cured copper foil or silver paste. Since the integrated surface probe method requires installing electrodes on only one side of the target CFRP structures, the method does not involve troublesome wire placement on inside surfaces of the composite tank structures. This enables electrical resistance change measurements on a CFRP composite tank without design changes to the target composite fuel tank.

Our previous paper adopted a four-probe method to detect a matrix crack in cross-ply laminates ${ }^{(19)}$. Residual electrical resistances at complete unloading and slopes at reloading are adopted to detect the existence of matrix cracking. In the present study, the matrix crack density is monitored using multiple electrodes with the electrical potential change method. Several electrodes are mounted on a CFRP rectangular specimen, and monitoring of the matrix crack density is performed for each segment between the electrodes. An FEA is performed to investigate the applicability of the monitoring of the matrix crack density using multiple electrodes, and experiments are also conducted to confirm the FEA.

\section{Principle of electrical resistance change method}

In CFRP laminates, carbon fibers have high electrical conductivity; the epoxy matrix is its insulator. The actual carbon fibers in a unidirectional ply are not straight. The curved carbon fibers are in contact with one another, producing a carbon fiber network within a ply. The contact-network produces non-zero electric conductivity even in the transverse direction. In the same way, the fiber-network produces non-zero electrical conductivity in the thickness direction in a ply. Electric conductivity in the transverse direction is lower than that in the fiber direction. Abry et al. ${ }^{(7)}$ and the authors' group ${ }^{(14)}$ experimentally found that the fraction of electrical conductivity in the transverse direction $\left(\sigma_{90}\right)$ relative to the fiber direction $\left(\sigma_{0}\right)$ is very small, and that the fraction of the electrical conductivity in the thickness direction $\left(\sigma_{t}\right)$ relative to the fiber direction is slightly smaller than that in the transverse direction. The results indicate that CFRP laminates have significantly strong orthotropic electrical conductivity.

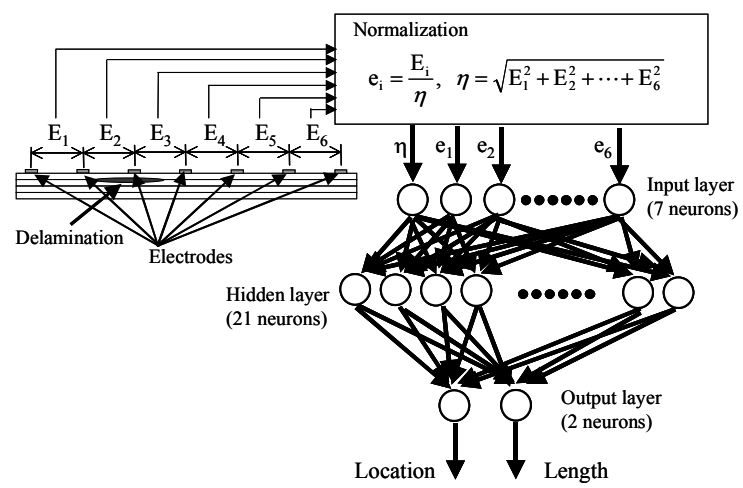

Fig. 1 Schematic representation of delamination identification method using the electric resistance change method with an artificial neural network

Figure 1 is a schematic representation of the delamination-monitoring system proposed by the authors ${ }^{(12)-(16)}$. Multiple probes of equally narrow spacing of approximately $35 \mathrm{~mm}$ are integrated on a single surface of the specimen. All the probes are placed on one side of the specimen. Usually, it is impossible to place electrodes and lead wires outside aircraft structures. The location of electrodes being only on one side is a model for placing electrodes inside a thin shell aircraft structure. The electrical resistance or electrical voltage change for each segment between adjacent electrodes is measured using a normal amplifier 
for conventional strain gages. Multiple point results are normalized and input into an artificial neural network. Delamination location and length are estimated from the measured results. The method is applied to a thin CFRP plate, and the delamination crack has been monitored with small error ${ }^{(12)-(16)}$.

In the previous paper ${ }^{(19)}$, a four-probe method was adopted to detect a matrix crack in CFRP cross-ply laminates. The outer two probes were used to apply electric current, and the inner two probes were used to measure the electrical voltage change. The four-probe method usually does not depend on the contact resistance at the probes, and the method is used for measurements of small changes in electrical resistance. In the previous paper, the matrix cracking was detected from the residual electrical resistance and slope of the reloading process.

\section{FEA}

\subsection{Analysis method}

FEA was performed with the commercially available finite element method (FEM) code ANSYS. Since a tension type specimen was used in the experiments, a two-dimensional type specimen model was adopted for FEA as shown in Fig. 2. The stacking sequence of the specimen laminate was $[0 / 90 / 0]_{\mathrm{T}}$. The length of the specimen was $200 \mathrm{~mm}$ and the thickness was $0.75 \mathrm{~mm}$; each ply had a thickness of $0.25 \mathrm{~mm}$. Six 2-mm-wide probes comprising copper electrodes were mounted on the single specimen surface in this finite element (FE) model. The outer two probes applied electric current, and the inner four probes measured the electric voltage; the locations were $x=-75,-45,-15$, $15,45,75 \mathrm{~mm}$.

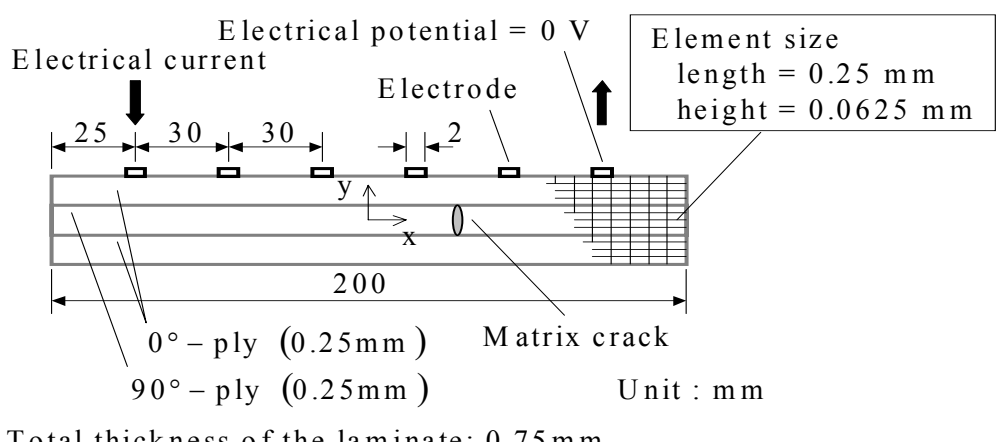

Fig. 2 Specimen of the FEA model

An automatic mesh division system installed in ANSYS was used. Four-node rectangular elements were adopted for the FEA; the length of each element was $0.25 \mathrm{~mm}$ and its approximate height was $0.0625 \mathrm{~mm}$. The total number of elements was approximately 9,600 .

A direct current of $I=50 \mathrm{~mA}$ was applied at one outer electrode and the electrical voltage of the other outer electrode was set to zero [V], and the inner four electrodes measured the electrical voltage $V$.

Orthotropic electrical conductivities used in the FEA were derived from previous experimental results ${ }^{(14)}$. In this study, a fiber volume fraction $V_{f}=0.62$ was employed. The electrical conductivity in the fiber direction $\left(\sigma_{0}\right)$ was $5500 \mathrm{~S} / \mathrm{m}$, the electrical conductivity in the transverse direction $\left(\sigma_{90}\right)$ was $204 \mathrm{~S} / \mathrm{m}$, and the electrical conductivity in the thickness direction $\left(\sigma_{t}\right)$ was $20.4 \mathrm{~S} / \mathrm{m}$.

The specimen in Fig. 2 was subjected to a tensile load in the horizontal direction. This caused matrix cracking in the middle $90^{\circ}$ ply between the surface $0^{\circ}$ plies. All FEM nodes of the candidate matrix cracks were doubly defined to represent matrix cracking. 
When a matrix crack forms, the doubly defined nodes on the target position separate to represent insulation of the electric current. For a matrix crack induced in the finite element (FE) model, the present study assumes the crack mouth is fully open after matrix cracking. Since tensile residual stress due to the curing of epoxy resin exists in the $90^{\circ}$ ply, a matrix crack mouth opens with the relief of the tensile residual stress. The FE model represents this crack opening. Spacing between adjacent matrix cracks is uniform in the FEA.

\subsection{Results and discussion}

First, a single matrix crack was induced in the FE model to investigate the effect of the location of a matrix crack compared to that of the location of an electrode. Figure 3 shows the results of the electric voltage change at the surface of the specimen for five locations of the matrix crack. The abscissa is the location measured from the center of the specimen and the ordinate is the electric voltage change from the initial value at the surface. The numbers adjacent to the curves are the locations of the matrix cracks. The electrodes applying the electric current are at $x=-75 \mathrm{~mm}$; the gray strip represents the positions of the electrodes.

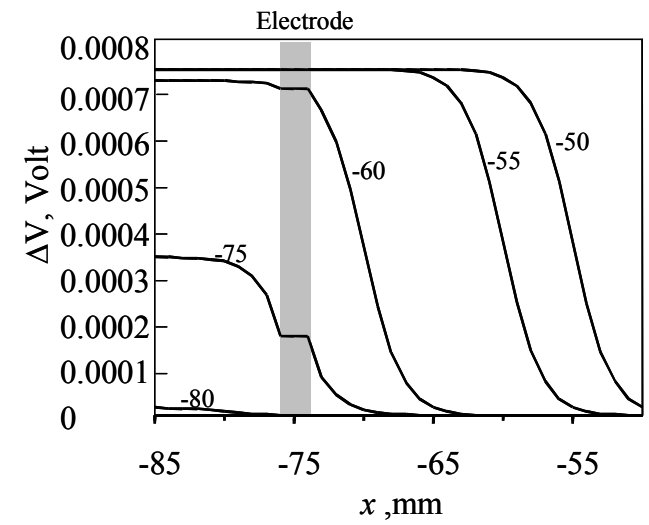

Fig. 3 Electric voltage change due to a matrix crack

(area near the electrode where electric current is applied)

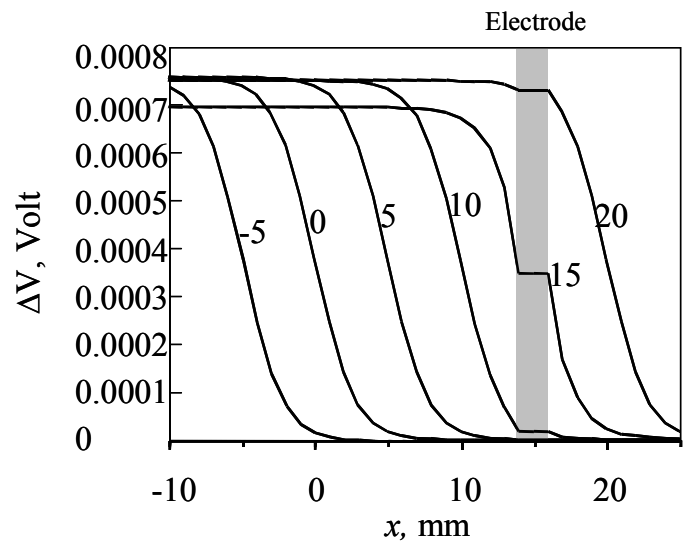

Fig.4 Electric voltage change due to a matrix crack

(around the center area)

In Fig. 3, the solid curve for $x=-80 \mathrm{~mm}$ represents a single matrix crack existing at $x=-80 \mathrm{~mm}$. The matrix crack is located outside of the two probes applying the electric current. Although a slight electric voltage increase is observed in the region left of $x=-80$ $\mathrm{mm}$, no voltage change is observed to the right of $x=-75 \mathrm{~mm}$. The solid curve for $x=-75 \mathrm{~mm}$ represents a single matrix crack located just under the electrodes applying the electric current. Although an electric voltage increase is observed at the electrode, there is no voltage 
increase in the region to the right of $x=-70 \mathrm{~mm}$. The other solid curves show the electric voltage increases greatly when the matrix crack is to the right of the electrodes. The size of the voltage increase has an upper bound that does not change with the location of the matrix crack when the crack is further than $15 \mathrm{~mm}$ from the electrodes.

Figure 4 shows the results of electric voltage changes around the center area of the specimen. The abscissa is the location and the ordinate is the electric voltage change at the specimen surface. The numbers adjacent to the curves are the location of the single matrix crack. In this figure, an electrode to measure electric voltage change is located at $x=15 \mathrm{~mm}$. Adjacent electrodes are located at $x=-15 \mathrm{~mm}$ and $x=45 \mathrm{~mm}$. The solid curves of $x=-5 \mathrm{~mm}, 0$ $\mathrm{mm}, 5 \mathrm{~mm}$ and $10 \mathrm{~mm}$ represent the voltage changes for a single crack located between the adjacent electrodes. For these cases, no voltage change is observed at the electrode for $x=10$ mm. For a matrix crack under the electrode $(x=15 \mathrm{~mm})$, the voltage at the electrode increases. For a single crack on the right $(x=20 \mathrm{~mm})$ of the electrode, the measured voltage rises to a maximum value. The upper limits of all curves indicate the voltage increase maintains its upper limit even for a crack exit further right than the electrode.

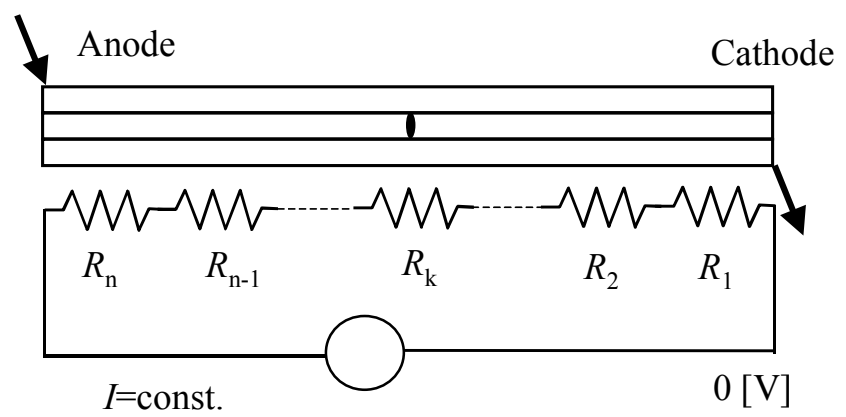

Fig. 5 Serially connected resistance model

Let us consider that the CFRP beam can be modeled as serially connected resistances as shown in Fig. 5. In this serially-connected-resistance model, a single matrix crack with a resistance $k$ in Fig. 5 can be modeled as a slight increase in resistance of $R_{\mathrm{k}}$. Since the constant electric current $I$ is applied in the specimen and the left end of $R_{\mathrm{n}}$ is kept at zero [V], the electrical voltage $\left(V_{\mathrm{i}}\right)$ of the $i{ }^{\text {th }}$ resistance from the right side is calculated easily as follows.

$$
V_{i}=\sum_{j=1}^{i} R_{j} I
$$

Eq. (1) indicates that a slight increase in $R_{\mathrm{k}}(1 \leq k \leq i)$ means a slight increase in the measured voltage. There is no increase in $V_{\mathrm{i}}$ for a single crack located on the anode electrode side of the $V_{\mathrm{i}}$ electrode. The voltage increase due to the single matrix crack is not the perfect shape of a step function, and the rising edge of the solid curve is not sharp. This is due to the orthotropic conductivity of CFRP.

This model implies that increases in voltage due to matrix cracks have no interaction between adjacent matrix cracks. This means the matrix crack density can be detected on a specific segment between electrodes when a constant electric current is applied to the CFRP specimen. To investigate the applicability of monitoring matrix crack density, many cases of multiple matrix cracks were analyzed in the present study.

Figure 6 shows all cases of matrix crack density used in the present study. The abscissa is the range over which multiple matrix cracks were generated. The ordinate is the matrix crack density. As shown in Fig. 6, eleven cases are calculated to investigate the applicability of the matrix crack density monitoring. A plot in Fig. 6 does not refer to a single FEA. The location of the matrix cracking area is changed at the fixed matrix crack density and distribution range. A total of $74 \mathrm{FE}$ analyses were performed for various locations, densities and distribution ranges of matrix cracking. 


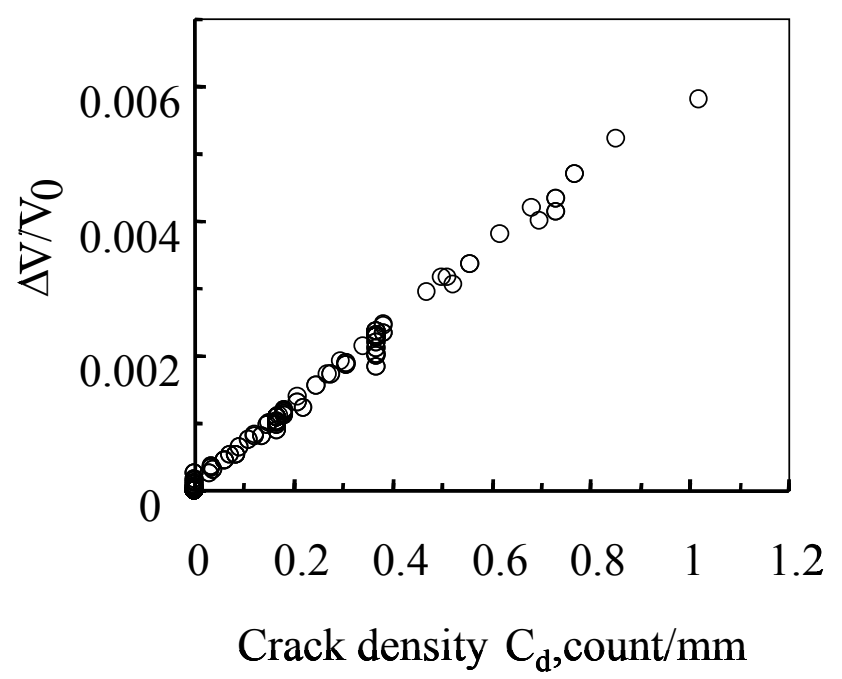

Fig. 6 Calculated electrical resistance changes for various matrix densities (Segment from $-75 \mathrm{~mm}$ to $-45 \mathrm{~mm}$ )

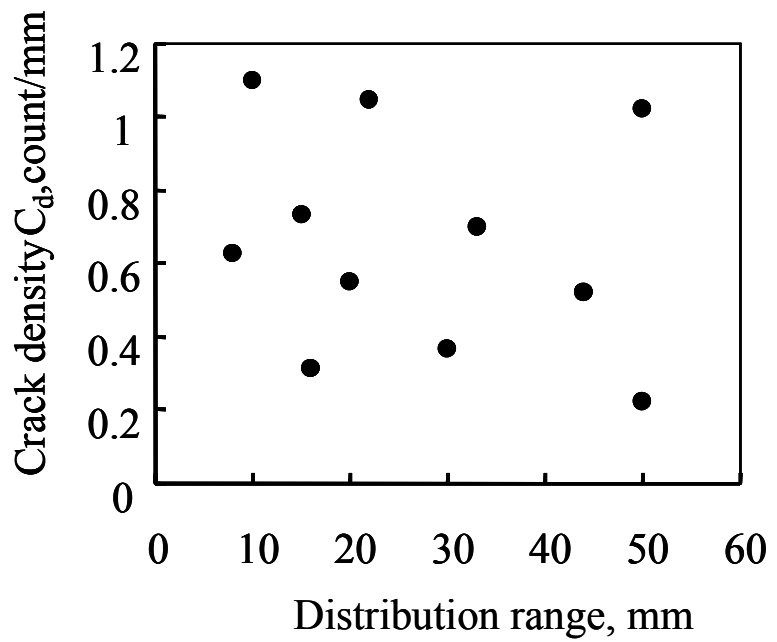

Fig. 7 Matrix crack aggregations used to investigate the applicability of the method for matrix crack density monitoring

Figure 7 shows the electrical resistance changes for the outermost segment near the anode electrode (segment from $-75 \mathrm{~mm}$ to $-45 \mathrm{~mm}$ ). The abscissa is the matrix crack density of the target segment, and the ordinate is the electrical voltage change ratio: the electrical voltage change between the target electrodes $(\Delta V)$ is divided by the initial electric voltage difference $\left(V_{0}\right)$. The results show the electrical resistance increases linearly with an increase in the density of matrix cracks. This means that an increase in the electrical voltage reveals the existence of matrix cracks and the size of the increase gives the density of the matrix cracks. This shows the applicability of monitoring matrix crack density with the electrical resistance change method. 


\section{Experimental investigation}

\subsection{Experimental method}

Unidirectional carbon/epoxy prepreg Mitsubishi Rayon TR380/G250 is used in the experiment. Cross-ply laminate with a stacking sequence of [0/90]s is adopted. The cross ply laminate is cured at $0.56 \mathrm{MPa}$ and $130^{\circ} \mathrm{C}$ for $90 \mathrm{~min}$ with a hot press method. After curing, the cross-ply laminate is cut into rectangular specimens of $245 \mathrm{~mm}$ length and 15 $\mathrm{mm}$ width. The thickness of each specimen is approximately $0.9 \mathrm{~mm}$. To prevent matrix cracking from machining flaws, the specimen edges are carefully polished with fine sandpaper.

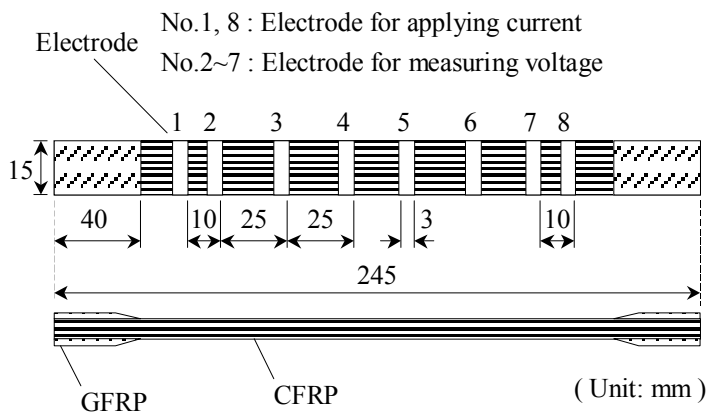

Fig. 8 Specimen configuration

Eight electrodes are mounted on the single surface of the specimen as shown in Fig. 8. The electrodes are produced after polishing the specimen surface to remove surface epoxy resin. The eight electrodes are made using silver paste. Silver paste is also used to attach lead wires to the electrodes. The two outermost electrodes (1 and 8) apply electric current and the inner six electrodes measure electric voltage changes. For the measurement of electrical voltage, an LCR meter made by Hioki Co. of type 3522-50 is used. The applied constant electric current is $10 \mathrm{~mA}$ and $1 \mathrm{kHz}$ alternating current is used for the measurement. An alternating current is chosen because it allows the CFRP to behave like a simple resistance without electrical capacitance. Moreover, the LCR meter has lower noise for alternating current. A strain gage is mounted at the center of the specimen to measure the applied strain. A tensile test is performed at $0.1 \mathrm{~mm} / \mathrm{min}$ loading speed. The matrix crack density is measured by means of a plastic film replica method during loading.

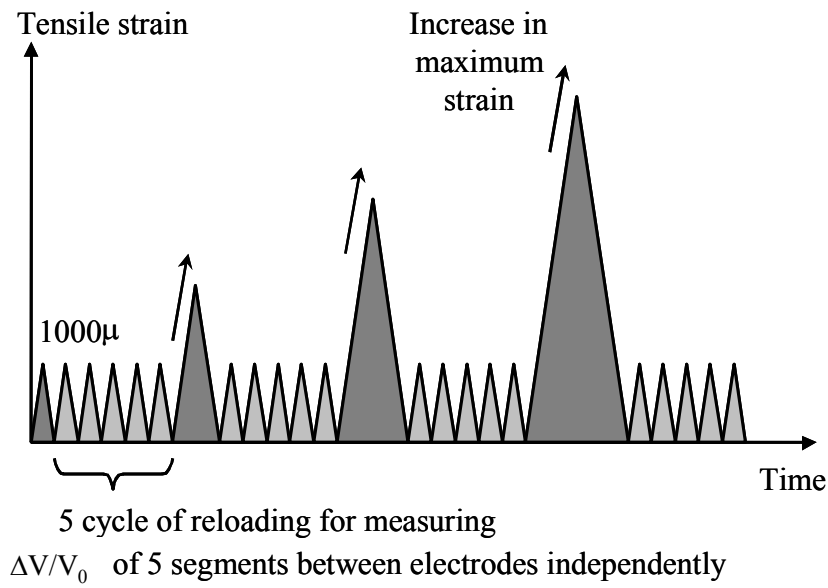

Fig. 9 Load-unload cycle schedule for experiments

Figure 9 shows the loading unloading schedule of the tensile test. Maximum loading is increased to $5000 \mu$. Before reloading, small loading cycles to $1000 \mu$ are applied to measure the slope at reloading. Five reloading cycles were performed to measure the electric 
voltage change slopes against the applied strain in the five segments between electrodes (\#2-3, \#3-4, \#4-5, \#5-6 and \#6-7).

\subsection{Results and discussion}

Figure 10 shows the results for various load-applied strains. The abscissa is the applied strain measured by means of a conventional strain gage, and the ordinate is the measured change in the electrical voltage difference of the segment between electrode \#4 and electrode \#5. The solid curves represent results at loading and the broken curves represent results at unloading. The voltage difference $\Delta V$ between electrodes $\# 4$ and $\# 5$ is divided by the initial voltage difference $V_{0}(=1.018 \mathrm{mV})$. In all other segments, the initial electrical voltage differences are a similar value.

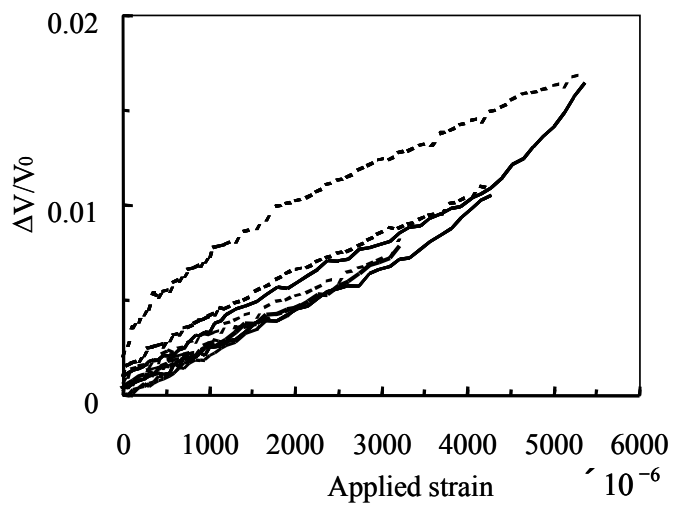

Fig. 10 Measured strain - electrical voltage difference curve

Figure 10 shows there is a linear relationship between $\Delta V / V_{0}$ and applied strain up to an applied strain of $3000 \mu$. For a strain more than $3000 \mu$, the electrical resistance increases rapidly with an increase in applied tensile strain. When the specimen is unloaded, the specimen has residual electrical voltage when the maximum applied strain exceeds $3000 \mu$. This is observed in all segments similarly.

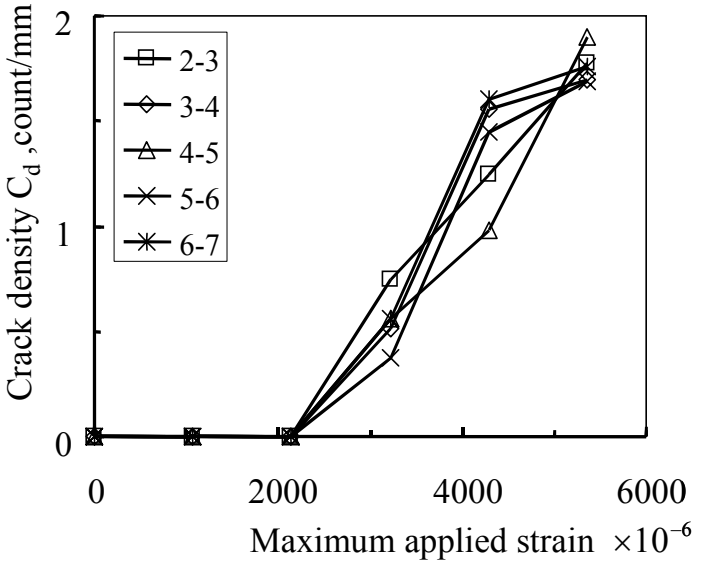

Fig. 11 Measured matrix crack density for all segments between electrodes

Figure 11 shows the measured matrix crack density obtained using the plastic film replica method on the specimen side surface. The abscissa is the maximum applied tensile strain and the ordinate is the measured matrix crack density for each segment. Figure 11 shows the matrix cracking starts at approximately $3000 \mu$. Since this is a uniform tensile test, the matrix crack densities in all segments between adjacent electrodes are similar, with only small differences observed among the segments. In Fig. 10, a rapid increase in electric voltage difference is observed from an applied strain of $3000 \mu$. Figure 11 shows the matrix 
cracking starts from an applied strain of $3000 \mu$. It can be concluded that the matrix cracking increases the electrical resistance and causes an increase in voltage difference. The residual electrical voltage difference is similarly observed over $3000 \mu$, as in our previous paper ${ }^{(19)}$.

Figure 12 shows the measured residual electrical voltage difference from the adjacent electrode under the complete unloading condition. The abscissa is the maximum applied strain of the cyclic loading and the ordinate is the electrical resistance under the complete unloading condition. The numbers 2-7 refer to electrode numbers. Except for segment \#5-6, every residual electric voltage difference seems to increase from a maximum applied strain of $2000 \mu$. The increase from $2000 \mu$, however, is not distinct in Fig. 12.

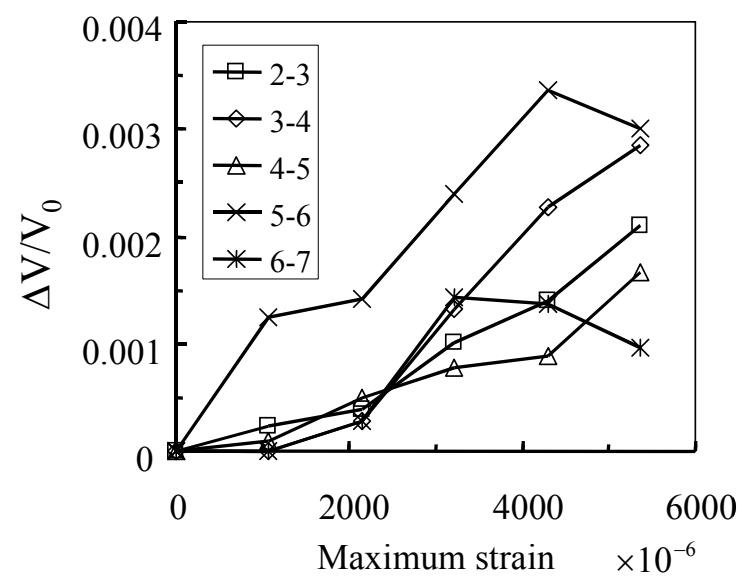

Fig. 12 Residual electrical resistance under unloading condition for each segment between electrodes

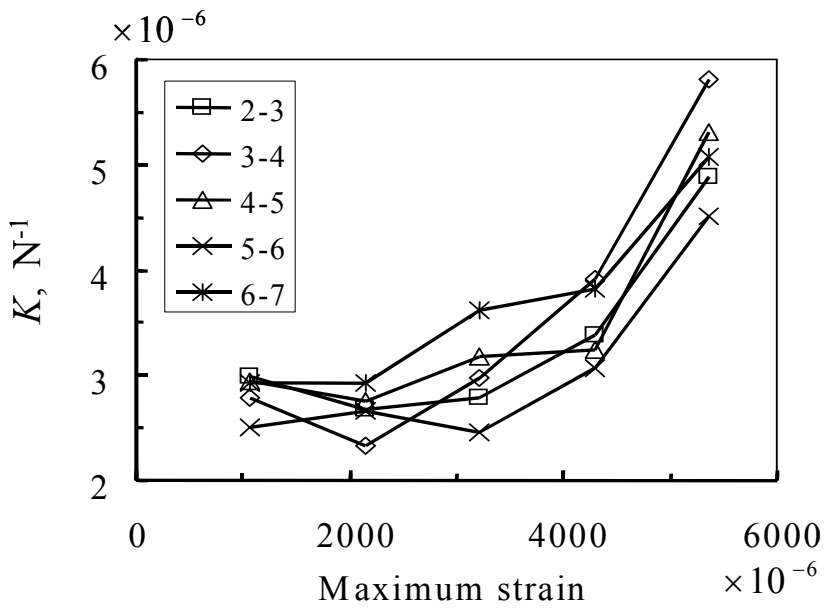

Fig. 13 Measured slope at reloading for each segment

Let us consider these results comparing with the matrix crack density. From Fig. 11 , the matrix crack density at a strain loading of $4200 \mu$ is obtained; segment \#6-7 has the highest crack density, segment \#3-4 has the second highest, segment \#5-6 has the third highest, segment \#2-3 has the fourth highest and segment \#4-5 has the lowest crack density. The lowest crack density is 1.0 and the highest crack density is 1.72 ; this means the highest number of cracks is 42 , in segment \#6-7, and the lowest number of cracks, in segment \#4-5, is 25 . This implies the difference in the number of cracks is not large.

From Fig. 12, the electrical voltage difference under the unloading condition after an applied strain of $4200 \mu$ can be observed; segment \#5-6 has the largest difference, segment \#3-4 has the second largest: segment \#2-3 has the third largest, segment \#6-7 has the fourth largest and segment \#4-5 has the smallest difference. Although the difference in the number of cracks is small, this result shows the residual electrical voltage difference under the 
unloading condition is not a good indicator of the matrix crack density for each segment. The voltage difference may be affected by crack closure at unloading; this crack closure may also be affected by adjacent cracks or the incline of the matrix cracks.

Figure 13 shows the measured slope at reloading between electrical resistance and applied load. The reloading slopes are measured between approximately $400 \mu$ and $800 \mu$ during the five cyclic loading to $1000 \mu$ : the definition of the slope is measured inclination at reloading from $800 \mu$ to $1000 \mu$. Hence the reloading slopes are measured when matrix cracks have reopened. The reloading slopes have small deviation and are a good indicator of matrix crack density. The abscissa is the segment number and the ordinate is the slope of the relationship between the electrical voltage difference and the applied strain. At an applied strain of $4200 \mu$, the steepest slope is obtained for segment \#3-4, the second steepest is for segment \#6-7, the third steepest is for segment \#2-3, the fourth steepest is for segment \#4-5 and the least steep is for segment \#5-6. Although the descending sequence of the slope values is a little different from the true matrix crack density, the differences among slopes are very small, and the variations in the slopes are similar to the distributions of the true matrix crack density.

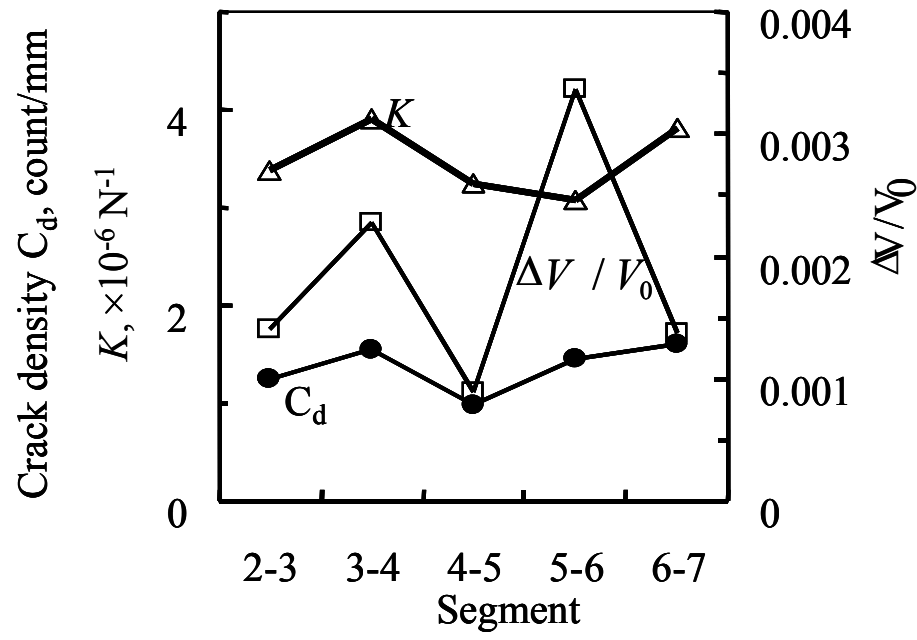

Fig. 14 Measured residual electrical resistances and reloading slopes after loading of a $4200 \mu$ strain.

Figure 14 shows the reloading slopes and residual electrical voltage differences after an applied strain of $4200 \mu$. The abscissa is the segments between electrode numbers and the left ordinate is the matrix crack density, and the reloading slope is also shown by the left ordinate. The right ordinate is the residual electrical voltage difference. The reloading slope is a good indicator in the monitoring of matrix crack density as it corresponds well with the true matrix crack density, while the residual electrical voltage difference does not.

The matrix crack density is measured by means of a visual observation of only a single edge of the specimen. Therefore, the matrix crack density does not always represent fully-grown matrix cracks. Since the number of matrix cracks is not great in the present study, the difference between the measured matrix crack density and that determined by the reloading slope appears very small.

\section{Conclusions}

The present paper deals with monitoring of the matrix crack density of CFRP laminates by means of electrical resistance changes. Multiple probes are mounted on a single surface of a CFRP plate. FEA was performed to investigate the possibility of monitoring the matrix crack density and an experimental investigation was also performed to confirm the FEA. The results obtained are as follows.

(1) Matrix cracks increase the electrical resistance only for the segment between electrodes 
in which the matrix cracks exist. There is no interaction between adjacent segments.

(2) An increase in matrix cracks is directly proportional to the electrical resistance of the segment where the matrix cracks are located.

(3) The experimental results show reloading slopes between applied load and electrical resistance are a good indicator of matrix crack density of the target segment between the electrodes.

\section{References}

(1) T.Aoki, T.Ishikawa, H. Kumazawa and Y.Morino, Cryogenic mechanical properties of $\mathrm{CF} /$ polymer composites for tanks of reusable rockets. Advanced Composite Materials,Vol.10, No.4, (2001), pp.349-356.

(2) K.Schulte and Ch. Baron, Load and Failure Analyses of CFRP Laminates by Means of Electrical Resistivity Measurements. Composites Science and Technology, Vol.36, No.1, (1989), pp.63-76.

(3) Chen P.W., and Chung D.D.L., Carbon Fibre Reinforced Concrete for Smart Structures Capable of Non-Destructive Flaw Detection. Smart Materials and Structures, No.2, Vol.1, (1993), pp.22-30.

(4) A.S.Kaddoour, F.A. Al-Salehi and S.T.S. Al-Hassani, Electrical Resistance Measurement Technique for Detecting Failure in CFRP Materials at High Strain Rate. Composites Science and Technology.,No.51, No.3, (1994), pp.377-385.

(5) X. Wang and D.D.L. Chung, Sensing Delamination in a Carbon Fibre Polymer-Matrix Composite During Fatigue by Electrical Resistance Measurement. Polymer Composites, Vol.18, No.6, (1997), pp.692-700.

(6) P.E.Irving and C. Thiagarajan, Fatigue Damage Characterization in Carbon Fibre Composite Materials using an Electric Potential Technique. Smart Materials and Structures.Vol.7, No.4, (1998), pp.456-466.

(7) J.C. Abry, S.Bochard, A.Chateauminois, M.Salvia and G. Giraud, In situ detection of damage in CFRP laminates by electric resistance measurements. Composites Science and Technology, Vol.59, No.6,(1999), pp.925-935.

(8) D.C. Seo and J.J.Lee, Damage Detection of CFRP Laminates using Electrical Resistance Measurement and Neural Network. Composite Structures. Vol.47, No.1-4,(1999), pp.525-530.

(9) I.Weber, P.Schwartz, Monitoring Bending Fatigue in Carbon-Fibre/Epoxy Composite Strands: a Comparison between Mechanical and Resistance Techniques. Composites Science and Technology, Vol.61, No.6, (2001), pp.849-853.

(10) J.B.Park., T.Okabe, N.Takeda and W.A.Curtin, Electromechanical modeling of unidirectional CFRP composites under tensile loading condition. Composites: Part A, Vol.33, No.2,(2002), pp.267-275.

(11) K.Ogi and Y.Takao, Characterization of piezoresistance behavior in a CFRP unidirectional laminate. Composites Science and Technology, Vol.65. No.2, (2005), pp.231-239.

(12) A.Todoroki, K.Matsuura and H.Kobayashi, Application of Electric Potential Method to Smart Composite Structures for Detecting Delamination. JSME International J., Series A, Vol.38, No.4, (1995), pp.524-530.

(13) A.Todoroki, Effect of number of electrodes and diagnostic tool for delamination monitoring of graphite/epoxy laminates using electric resistance change. Composites Science and Technology, Vol.61, No.13, (2001), pp.1871-1880.

(14) A.Todoroki, M.Tanaka, and Y.Shimamura, Measurement of Orthotropic Electric Conductance of CFRP laminates and Analysis of the Effect on Delamination Monitoring with Electric Resistance Change Method, Composites Science and Technology, Vol.62, No.5, (2002), pp.619-628. 
(15) A.Todoroki, Y.Tanaka, and Y.Shimamura, Delamination monitoring of graphite/epoxy laminated composite plate of electric resistance change method. Composites Science and Technology. Vol.62, No9, (2002), pp.1151-1160.

(16) A.Todoroki, Y.Tanaka, and Y.Shimamura, Identifying Delamination in Cross-ply and Quasi-isotropic Beams of CFRP by a Standardized Electrical Resistance Method, Polymers \& Polymer Composites.Vol.12, No.1, (2004), pp.75-85.

(17) A.Todoroki, Electric Resistance Change Method for Cure/Strain/Damage Monitoring of CFRP Laminates. Key Engineering Materials, Vol.270-273, (2004), pp.1812-1820.

(18) A.Todoroki and J.Yoshida, Electrical resistance change of unidirectional CFRP due to applied load, JSME International J., Series A, Vol.47,No.3, (2004),pp.357-364.

(19) A.Todoroki, K.Omagari, Y.Shimamura and H.Kobayshi, Matrix crack detection of CFRP using electrical resistance change with integrated surface probes, Composites Science and Technology, Vol.66, No.11-12, (2006), pp.1539-1545 